\title{
Serious allergic reactions following tuberculin skin tests
}

Published at www.cmaj.ca on June 15, 2005.

Reason for posting: Tuberculosis poses an enormous health burden worldwide, and tuberculin skin tests, which are simple and sensitive, are widely used to screen for the infection. Local hypersensitivity reactions are expected, intended and, in fact, an expression of what the test is supposed to measure. Recently, however, Health Canada has warned of serious allergic reactions, including anaphylaxis, in some recipients.

The test: Mantoux tuberculin skin tests are often administered to those who may be exposed to tuberculosis infection, including people from endemic areas, health care workers and others who work in long-term care facilities, group homes, drug treatment centres, prisons and shelters for the homeless. People who are immunosuppressed (e.g., patients with HIV infection, diabetes or renal failure) are also often tested, because they are at greater risk of developing the active form of tuberculosis.

To perform the test, 5 tuberculin units of purified protein derivative (PPD) of Mycobacterium tuberculosis is injected intradermally, often in the volar skin of the forearm. A delayed hypersensitivity reaction usually starts within 24 hours, reaching its peak between 48 and 72 hours, which is when the test result is read. Characteristically, a circular area of erythema occurs around a more central area of induration. (Guidelines for interpreting the test result are covered elsewhere. $\left.{ }^{2}\right)$ In 1\%-2\% of positive test results, blistering or even local necrosis may occur, but this is usually self-limited. ${ }^{2}$ Local reactions such as regional lymphangitis and adenitis may also occur on rare occasions.

Health Canada now reports systemic hypersensitivity reactions occurring in some people after the test, including anaphylaxis, urticaria, angio- and other edema, and throat swelling. Although the Health Canada advisory reports few clinical details, these reactions appear to have occurred in at least some patients not previously exposed to the test.

Although such adverse events are probably underreported once a product is marketed, these serious reactions seem to occur at a rate of about 1 per million doses dispensed. This rate is similar to anaphylaxis rates typically reported for vaccines (1.1-3.1 per million distributed doses). ${ }^{3}$ In the past 12 years, 26 serious reactions were reported worldwide, including 9 in Canada.

What to do: Tuberculosis is a common and deadly disease, and targeted screening allows early recognition and the initiation of appropriate management. Serious adverse reactions to the test are rare, but patients should be warned that they can occur. The test should be administered only in settings where trained staff are prepared to deal with anaphylactic reactions and epinephrine (1:1000) is available. Patients receiving the injection should be observed for at least 15 minutes to monitor for serious adverse reactions, and warned to seek medical attention promptly if any delayed reactions occur.

\section{Elham Youssef}

Resident

Department of Family Medicine

University of Ottawa

Ottawa, Ont.

Eric Wooltorton

CMAF

\section{References}

1. Sanofi Pasteur Limited, Health Canada's Health Products and Food Branch, Public Health Agency of Canada. Risk of serious allergic reactions following Tubersol [tuberculin purified protein derivative (Mantoux)] administration [Dear Health Care Professional letter]. Ottawa: Health Canada; 2005. Available: www.hc-sc.gc .ca/hpfb-dgpsa/tpd-dpt/tubersol_hpc_e html (accessed 2005 Jun 8).

2. Menzies D, Nan Tannenbaum T, FitzGerald JM. Tuberculosis: 10. Prevention. CMAF 1999;161(6):717-24.

3. National Advisory Committee on Immunization. Canadian immunization guide. 6th ed. Ottawa: Health Canada; 2002. p. 14.

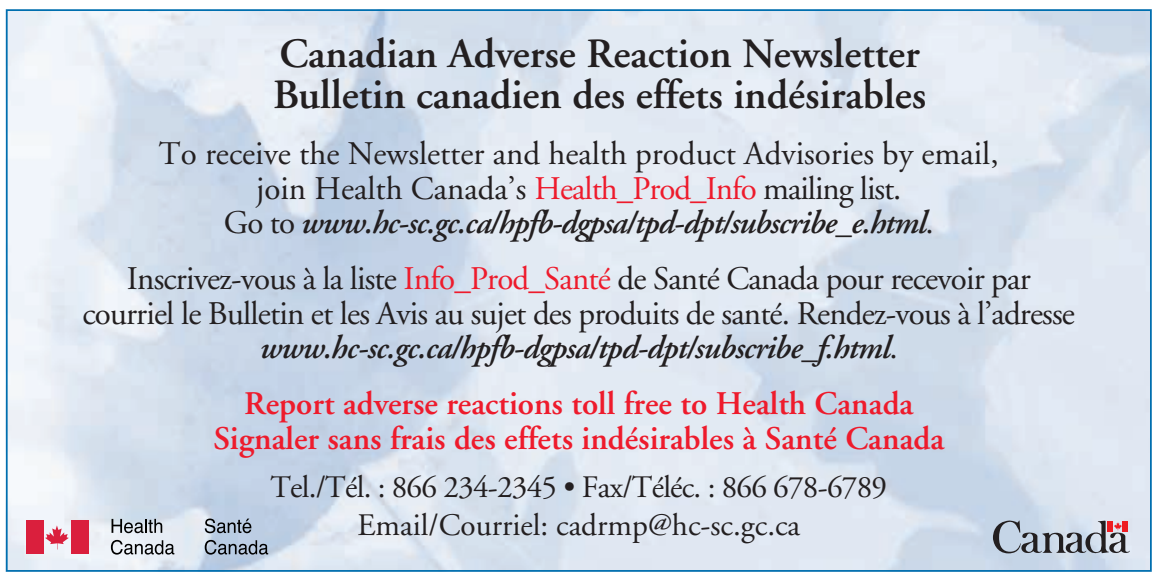

